\title{
Antibiotic Sensitivity and Histopathological Study of Clostridium perfringens Associated with Necrotic Enteritis in Poultry
}

\author{
Prerana R. Shelke, Mrunalini M. Pawade*, Prashant P. Mhase, \\ Prajwalini V. Mehere and Jyotika D. Sangle
}

Department of Veterinary Microbiology, KNP College of Veterinary Science, Shirwal, India

*Corresponding author

\begin{tabular}{|l|}
\hline Ke y w o r d s \\
Necrotic enteritis, \\
Poultry, \\
Clostridium \\
perfringens, \\
Antibiotic \\
sensitivity test, \\
Histopathology \\
\hline Article Info \\
\hline $\begin{array}{l}\text { Accepted: } \\
\text { 26 October } 2018 \\
\text { Available Online: } \\
\text { 10 November } 2018\end{array}$ \\
\hline
\end{tabular}

A B S T R A C T

Necrotic enteritis (NE) is sporadic disease of avian species which has been reported from most of the areas of world in which poultry are reared. The causative agent of necrotic enteritis is Clostridium perfringens, a gram-positive anaerobe. The aim of this study was isolation of $C$. perfringens from NE cases along with antibiotic sensitivity study of the isolates and histopathology of the NE suspected intestine. For the present study, total 50 samples were collected and inoculated in Robertson's cooked meat media for initial enrichment followed by streaking on clostridium agar with TSC supplements and on egg yolk agar. After anaerobic incubation for $24 \mathrm{hr}$, black coloured colonies were visualized on clostridial agar (with TSC supplement) and opalescent colonies were seen on egg yolk agar. Further identification of $C$. perfringens was done by microscopical appearance and biochemical characterization. Total $35(70 \%)$ samples out of 50 samples were collected and tested for the presence of $C$. perfringens. These isolated and pure colonies of $C$. perfringens were further examined for antibiotic sensitivity test. Histopathological examination of intestine of $\mathrm{NE}$ affected bird revealed, necrosis of villi, infiltration of heterophils, macrophages and deposition of variable number of fibrinous shreds and amorphous connective tissue which is one of the indicative findings of NE in poultry. Further study concluded RCMM as one of the best medium for enrichment of $C$. perfringens. Antibiotic sensitivity study of $C$. perfringens isolates was found that amoxicillin was the drug of choice for NE in poultry. This paper also describes the gross and microscopical important features of the intestine of NE due to C. perfringens.

\section{Introduction}

Poultry is one of the fastest growing and profitable segments of the agricultural sector in India with around eight percent growth rate per annum. India ranks 3rd in egg production and 7 th in chicken meat production in the world (Watt Executive Guide, 2015).
Clostridium perfringens is one of the most important food-borne pathogen of humans and animals causing both histotoxic diseases and intestinal infections. It is Gram-positive, anaerobic, straight rod, spore former, found in the soil, dust, sewage, marine sediments and in the gastro-intestinal tract of humans and animals. Based on the production of four 
major lethal toxins i.e. alpha $(\alpha)$ beta $(\beta)$, epsilon ( $\varepsilon$ ) and iota (i), $C$. perfringens is divided into five major toxinotypes (A-E) (Yadav et al., 2016). All C. perfringens strains are pathogenic for animals, only type A and $\mathrm{C}$ strains are found harmful to humans (Guran et al., 2013). C. perfringens associated with NE mainly produce type A and type C toxinotype (Shojadoost et al., 2010). Type A which mainly produces alpha toxin, has lecithinase activity and hydrolyzes lecithin into phosphorylcholine and diglyceride and causes pathologic changes in tissues (Kalender et al., 2005).

Necrotic enteritis is an important clinical disease produced by $C$. perfringens that affects the poultry industry worldwide causing serious economic loss, about of two-dollar billions/year. This disease is characterized by severe necrosis of the small intestine mucosa in the proximal jejunum region and it is associated with high mortality rates. On the other hand, subclinical disease leads to a decreased performance, due to the extensive mucosal damage (Llanco et al., 2012). In this study, the isolation, antimicrobial susceptibility and histopathology of $C$. perfringens strains isolated from chickens with necrotic enteritis were determinate.

\section{Materials and Methods}

A total of 50 intestinal samples which showed signs of enteritis were collected aseptically from poultry farm. Intestinal samples were collected from suspected cases of birds with enteritis referred for post mortem cases at Department of Pathology, KNPCVS, Shirwal and from Omega laboratory, Lonand, dist., Satara for this study. All samples were collected aseptically in a sterile container and quickly transported to the laboratory in icecooled containers. Processing of the collected samples was done as soon as samples received to the department (Ezatkhah et al., 2016).

\section{Isolation of $C$. perfringens}

All the samples were inoculated in Robertson's cooked meat media (RCMM) and in Fluid thioglycolate broth for initial enrichment. Inoculated broths were heated at $80^{\circ} \mathrm{C}$ for 10 minutes to destroy vegetative form of organisms followed by anaerobic incubation using anaerobic jars with anaerogas pack at $37^{\circ} \mathrm{C}$ for $24-48$ hours. After incubation a loopful of broth culture was further streaked onto selective agar and egg yolk agar plates for cultivation of Clostridium perfringens. Isolation of bacterial pathogens was done as per the standard methods described in Bergey's Manual of Systematic Bacteriology, 1986.

\section{Selective plating for growth of $C$. perfringens}

For isolation of $C$. perfringens, a loopful culture of RCMM broth culture was streaked on perfringens agar base (T.S.C Supplement) plate and incubated anaerobically in jar with anaerogas pack at $37^{\circ} \mathrm{C}$ for 24 - 48 hours.

Plates were observed for growth of Clostridium perfringens. After incubation the shape, size and type of colonies on agar were recorded.

Further confirmation of the isolates was done by streaking on egg yolk agar for lecithinase production followed by Gram's staining of the isolates. Pure cultures thus obtained were further processed for conventional biochemical characterization.

\section{Identification of $C$. perfringens}

After isolation, identification of $C$. perfringens was done by using Biochemical test such as catalase test, oxidase test and motility test (Hanging drop test). 
Antibiotic sensitivity test of bacterial isolates

Each of the bacterial isolate obtained from the samples of intestine of necrotic enteritis from suspected poultry birds were subjected to In Vitro antibiotic sensitivity testing by disc diffusion technique as per the method of Bauer et al., (1966). The commercially available antibiotic discs and interpretative chart of zone of inhibition were used from HiMedia Laboratories Ltd., India. The antibiotics and its concentrations used for this test were amoxicillin $(10 \mu \mathrm{g})$, ampicillin (10 $\mu \mathrm{g})$, amikacin $(30 \mu \mathrm{g})$, cloxacilline $(1 \mathrm{mcg})$, enrofloxacin $(10 \mu \mathrm{g})$, gentamycin $(10 \mathrm{mcg})$, oxytetracycline (30 mcg), penicillin (10 units), streptomycin $(10 \mu \mathrm{g})$.

\section{Histopathology of NE suspected intestine}

The tissue samples of intestine were collected in 10 percent Neutral buffered formalin (NBF) and processed by routine paraffin embedding method described by Chauhan (1995). The tissue section of 4-6 microns were stained with routine Hematoxylin $(\mathrm{H})$ and Eosin $(\mathrm{E})$ method suggested by Chauhan (1995).

\section{Results and Discussion}

Isolation and identification of $C$. perfringens from poultry

The $C$. perfringens isolated from $\mathrm{NE}$ suspected intestine were identified based on morphological, cultural and biochemical characteristics. After enrichment, total 35 samples showed heavy turbidity with gas production in RCMM, turning the meat particles pink in color along with foul odor, while FTG broth did not show any growth (Plate No.1). The present study showed RCMM under anaerobic condition was the best medium for isolation of $C$. perfringens from NE suspected samples.
RCMM was the found to be best medium for enrichment of $C$. perfringens as described by Das et al., (2008) and Nyrah et al., (2017) used RCMM for initial enrichment of $C$. perfringens from necrotic enteritis suspected broiler chicken. Agarwal et al., (2009) studied on $C$. perfringens and for the same they used thioglycolate enrichment medium for initial isolation.

\section{Identification of $C$. perfringens}

All the 35 positive broths which were streaked on perfringens agar with tryptose sulphite cycloserine (TSC) supplements, showed rough and black color colonies with sulphite reduction (Figure 2). Pure colonies from perfringens agar plates were selected and streaked on egg yolk agar medium, which showed characteristic diffused opalescence due to lecithinase activity of alpha toxins (Figure 3). Similar types of colonies were observed by Salah-Eldin et al., (2015) and Keyburn et al., (2010) of C. perfringens on egg yolk agar and clostridial agar with TSC supplement. Further morphological examination revealed Gram- positive, short and plumpy rods with blunt ends (Figure 1) which correlated well with the reports of Hafez et al., (2011) and Khairy et al., (2013).

On biochemical examination, the organisms failed to produce purple color on filter paper containing oxidase reagent and proved that the organisms were oxidase negative. The catalase test revealed none of the isolates produced effervescence and hence were catalase negative. On examination of isolates for motility by hanging drop preparation, it was proved that all the organisms were non-motile. Effat et al., (2007) and Mostafa et al., (2016) had reported $C$. perfringens isolates were found to be negative for oxidase and catalase.

In all total 35(70\%) samples were found positive for $C$. perfringens from 50 diseased 
(NE suspected) samples which were collected from NE suspected poultry birds.

Overall antibiotic sensitivity profiling of the 35 confirmed $C$. perfringens isolates, indicated that, amoxicillin was most effective against $30(85.71 \%)$ isolates of $C$. perfringens tested followed by ampicillin $25(71.42 \%)$ and oxytetracycline $20(42.85 \%)$. Total 18 $(51.42 \%)$ isolates were intermediate sensitive to enrofloxacin followed by gentamycin and streptomycin which were intermediate sensitive against $15(42.85 \%)$ and $10(28.37 \%)$ isolates respectively. Out of 35 isolates, 28 $(80 \%)$ were found resistant to penicillin, followed by $25(71.42 \%)$ to cloxacillin, 22 $(62.85 \%)$ to amikacin (Figure 4).

These findings were closely similar to the Llanco et al., (2012) found that the antibiotic like amoxicillin, amoxicillin-clavulanic acid, cefoxitin, chloramphenicol, enrofloxacin, metronidazole and penicillin streptomycin showed susceptibility towards isolates of $C$. perfringens. Martel et al., (2004) reported that the antibiotic avilamycin and amoxicillin showed good activity, while all isolates were resistant to high concentrations of flavomycin. Along with this Agarwal et al., (2009) found highest sensitivity to fluroquinolones Viz. ciprofloxacin (93.3\%) and enrofloxacin (86.6\%) followed by chloramphenicol $(76.6 \%)$, oxytetracycline (70\%), co-trimoxazole $(63.3 \%)$ cefatoxime (40.0\%), gentamicin (36.6\%), ampicillin $(33.3 \%)$ and colistin (13.3\%) and all the isolates showed highly resistant to streptomycin and neomycin (100\%). Tansuphasiri et al., (2005) and Singh et al., (2013) observed that $C$. perfringens isolates which were obtained from different sources showed resistance to tetracycline (28-78\%), ceftriaxone (1-2.5\%), chloramphenicol (3\%) and penicillin $(9 \%)$. The variation in the antimicrobial pattern might be due to indiscriminate use of these antibiotics as feed additive and prophylaxis as well as therapeutic agent in poultry industry in India.

\section{Histopathology of NE suspected intestine gross lesions}

It was observed that the intestine was having necrotic lesions of variable size, shape and consistency. These were small pin point dark in color, some were uneven shaped with irregular margins and covered with tenacious diptheric membranes, slightly elevated above the mucosa of intestines. Some of the lesions were blackish in color.

\section{Histopathological examination}

Histopathological examination revealed necrosis of villi, infiltration of heterophils, macrophages and deposition of variable number of fibrinous shreds and amorphous connective tissue. Amorphous material also includes dead cells and debris present around the mucosa and submucosa, in some of the cases it was extended up to muscularis layer (Figure 5).

Figure.1 Microscopic appearance of $C$. perfringens in Gram's stain

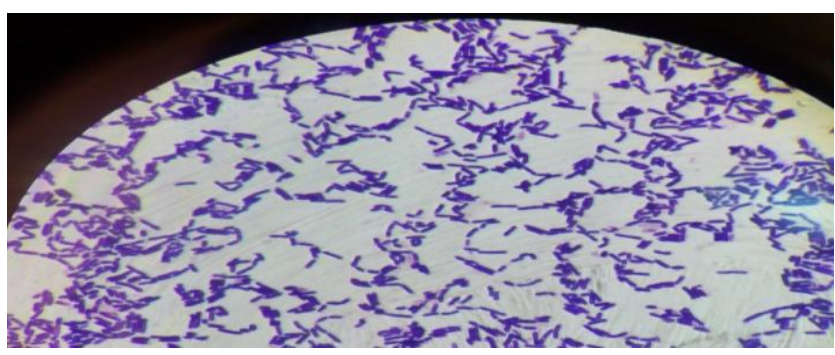


Figure.2 Black colonies of $C$. perfringens on clostridial agar with TSC supplement

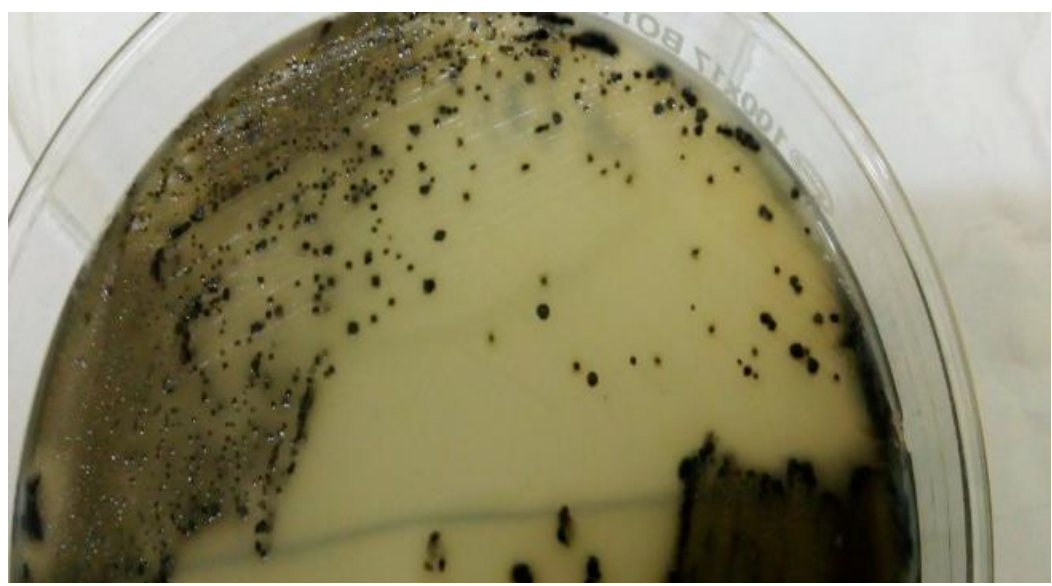

Figure.3 Opalescent colonies of $C$. perfringens on egg yolk agar



Figure.4 Antibiotic sensitivity pattern of $C$. perfringens isolates

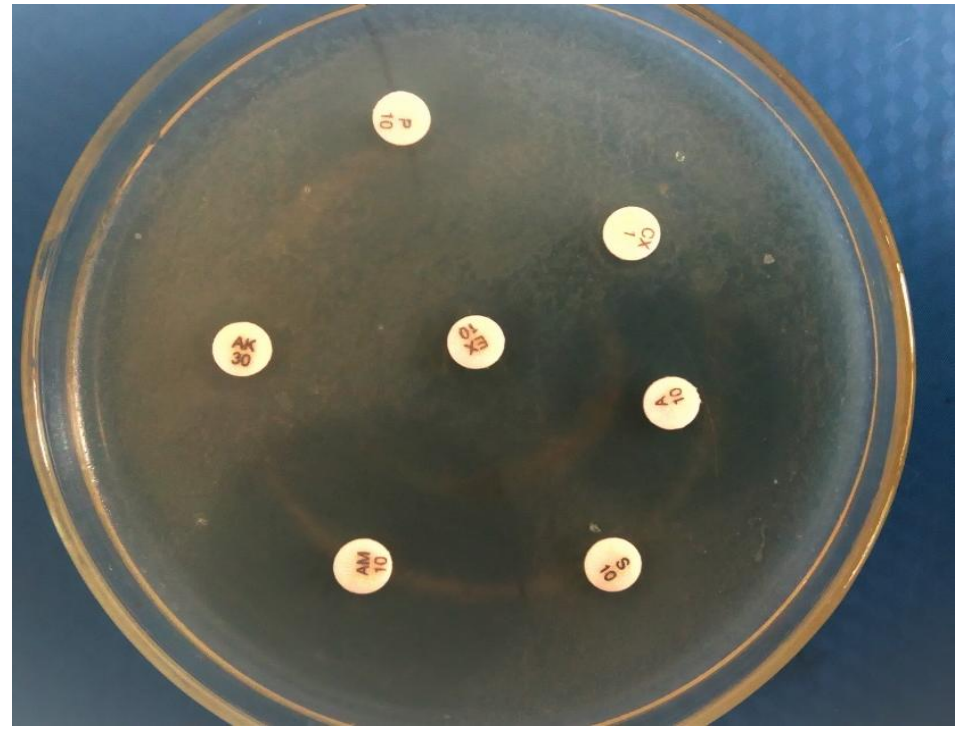


Figure.5 Histopathological examination showed destruction of villi

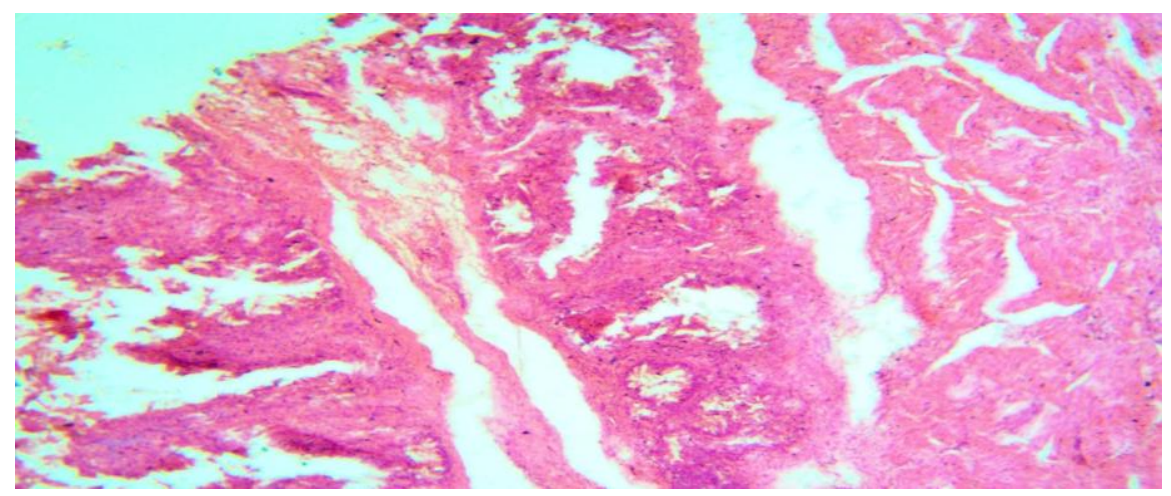

These findings were in agreement with some authors like Balachandran et al., (2018) and Brady et al., (2010) reported that on necropsy the mesenteric vessels were engorged with blood and intestinal lumen contained bile stained fluid and granular debris. The mucosa was covered with firmly adhering yellowish brown diphtheritic membrane giving a dirty Turkish towel appearance. On histopathology of the duodenal and jejunal sections showed necrosis of villous tips or entire villi, down to the crypts, with many large bacilli in this necrotic and cellular debris. Lyhs et al., (2012) recorded that the histopathology confirmed a severe necrosis by the affecting the tips of the villi or the entire villi, together with a demarcation zone of inflammatory cells, degenerated epithelial cells, fibrin, and colonies of clostridia-like, rod-shaped bacteria. However, the crypts were usually intact.

In conclusion, the overall incidence of necrotic enteritis due to $C$. perfringens was found to be 70 percent. RCMM was found to be the best medium than FTG for enrichment of $C$. perfringens. $C$. perfringens isolates was found sensitive to Amoxicillin, Ampicillin, Oxytetracycline followed by Enrofloxacin and higher percentage of resistant was found towards Penicillin, Cloxacillin and Amikacin. Histopathological examination of the intestine from NE suspected birds, confirmed the occurrence of $\mathrm{NE}$ in poultry. This paper describes the gross and microscopical important features of the intestine of NE due to $C$. perfringens.

\section{Acknowledgment}

I would like to thanks my college associate dean Dr. A. S. Ranade, KNP College of Veterinary Science, Shirwal.

\section{References}

Agarwal A., Narangi G., Rakhn N., Mahajan N. and Sharmn A. (2009). In vitro lecithinase activity and antibiogram of Clostridium perfringens isolated from broiler chickens. Haryana Vet. 48:8184.

Balachandran P., Srinivasan P., Balasubramaniam GA., Sivaseelan S. and Murthy TRGK (2018). Prevalence and predisposing factors in spontaneous cases of necrotic enteritis in cage reared commercial layer chicken. Adv. Anim. Vet. Sci. 6(3): 113-120.

Bauer A.W., Kirby W.M.M., Shernis J.C. and Turck M. (1996). Antibiotic Susceptibility testing by standardized single disc method. Am.J.Clin.Pathol. 45:493-496.

Bergey's Manual of Systemic Bacteriology, (1986). 
Brady J, Hernandez-Doria J. D., Bennett C., Guenter W., House J. D. and RodriguezLecompte J. C. (2010). Toxinotyping of necrotic enteritisproducing and commensal isolates of Clostridium perfringens from chickens fed organic diets. Avian Pathology 39(6): 475-481.

Chauhan H.V.S. (1995). Veterinary Clinical and laboratory Diagnosis.3rd Edn, Jaypee Publishers, New Delhi.

Das A., Mazumder Y., Dutta B., Shome B.R., Bujarbaruah K.M., and Kumar A. (2008). Clostridium perfringens Type A from Broiler Chicken with Necrotic Enteritis. International Journal of Poultry Science 7 (6): 601-609.

Effat M. M., Abdallah Y. A., Soheir M. F. and Rady M. M. (2007). Characterization of Clostridium perfringens field isolates, implicated in necrotic enteritis outbreaks on private broiler farms in Cairo, by multiplex PCR. African Journal of Microbiology Research. 029-032.

Ezatkhah M., Alimolaei M., Shahdadnejad N. (2016). The Prevalence of netB Gene in isolated Clostridium perfringens From Organic Broiler Farms Suspected to Necrotic Enteritis. International Journal of Enteric Pathogens 4(3): e35667.

Guran H. S. and Oksuztepe G.2013. Detection and typing of Clostridium perfringens from retail chicken meat parts. Letters in Applied Microbiology 57, 77-82.

Hafez Mohamed Hafez (2011) Enteric

Diseases of Poultry with Special Attention to Clostridium perfringens. Pak Vet J. 31(3): 175-184.

Kalender Haken.2005. Isolation of Clostridium perfringens from Chickens and Detection of the Alpha Toxin Gene by Polymerase Chain Reaction (PCR). Turk J Vet Anim Sci 29,847-851.
Keyburn A. L., Bannam T. L., Moore R. J. and Rood J. I. (2010). NetB, PoreForming Toxin from Necrotic Enteritis Strains of Clostridium perfringens. 2: 1913-1927.

Khairy E. A, Dorgham S. M., Bakry M.A and Hakim A.S. (2013). Molecular Diversity of Alpha Toxin Produced by Clostridium perfringens Strains Causing Avian Necrotic Enteritis. World Applied Sciences Journal 21 (1): 15-20.

Llanco L.A., Nakano V., FERREIRA A.J.P. AND Avila-campos M.J.2012. Toxinotyping and antimicrobial susceptibility of Clostridium perfringens isolated from broiler chickens with necrotic enteritis. International Journal of Microbiology Research Vol 4(7).

Llanco L.A., Viviane nakano, Ferreira A.J.P. and Avila-campos M.J. (2012). Toxinotyping and antimicrobial susceptibility of Clostridium perfringens isolated from broiler chickens with necrotic enteritis. International Journal of Microbiology Research, 4(7).

Lyhs U., (2013). Characterization of Clostridium perfringens isolates from healthy turkeys and from turkeys with necrotic enteritis. Poultry Science.92(7):1750-1757.

Martel A., Devriese L. A., Cauwerts K., De Gussem K., Decostere A., and Haesebrouck F. (2004). Susceptibility of Clostridium perfringens strains from broiler chickens to antibiotics and anticoccidials. Avian Pathology.33(1): 3-7.

Mostafa A. H., El-ShahatAbdeen E. and Abou-Hadeed M. G. (2016). Multiplex PCR and Detection of netB Gene of Clostridium perfringens from Broilers with Necrotic Enteritis. Asian Journal 
of Animal and Veterinary Advances. ISSN 1683-9919.

Nyrah Q., Wani S.A., Nazir N., Rasool S., Beigh Q., Kashoo Z.A., Hussain I., Qureshi S. and Ali R. (2017). Clostridium perfringens Type A from Broiler Chicken with Necrotic Enteritis in Kashmir Valley, India. Int. J. Curr. Microbiol. App. Sci 6(12): 2443-2453.

Salah-Eldin A. E., Fawzy E. H., Aboelmagd B. A., Ragab E. A. and Shaimaa B., (2015). Clinical and Laboratory Studies on Chicken Isolates of Clostridium perfringens in ElBehera, Egypt. J. World's Poult. Res. 5(2): 21 28.

Shojadoost, B., Peighambari S.M. and Nikpiran, (2010) Isolation, identification, and antimicrobial susceptibility of Clostridium perfringens isolates from acute necrotic enteritis of broiler chickens.
International Journal of Veterinary Research 4(3): 147151.

Singh R.V. and Bist B. (2013). Antimicrobial Profile of Clostridium perfringens Isolates from Dairy Products. Journal of Animal Research.3(2):147-151.

Tansuphasiri U., Matra W. and Sangsuk L. (2005). Antimicrobial resistance among $C$. perfringens isolated from various sources in Thailand. Southeast Asian Journal of Tropical Medicine and Public Health, 36(4).

Watt Executive Guide, (2015). Poultry Trends. www.wattagnet.com

Yadav J. P., Das S. C., Dhaka P.,Vijay D., Kumar M., Chauhan P., Singh R., Dhama K., Malik S.V.S and Kumar A. 2016. Isolation, Genotyping and Antibiogram Profile of Clostridium perfringens Isolates Recovered from Freshwater Fish and Fish Products from Kolkata Region. Journal of pure and applied microbiology. Vol. 10(4), 2807-2814.

\section{How to cite this article:}

Prerana R. Shelke, Mrunalini M. Pawade, Prashant P. Mhase, Prajwalini V. Mehere and Jyotika, D. Sangle. 2018. Antibiotic Sensitivity and Histopathological Study of Clostridium perfringens Associated with Necrotic Enteritis in Poultry. Int.J.Curr.Microbiol.App.Sci. 7(11): 3159-3166. doi: https://doi.org/10.20546/ijcmas.2018.711.363 